\title{
Chemotherapy and Hepatic Steatosis: Impact on Postoperative Morbidity and Survival after Liver Resection for Colorectal Liver Metastases
}

\author{
Jan C. Mahlmann ${ }^{\mathrm{a}}$ Thomas C. Wirth ${ }^{\mathrm{b}}$ Björn Hartleben ${ }^{c}$ Harald Schrem ${ }^{\mathrm{d}}$ \\ Jens F. Mahlmann ${ }^{\mathrm{e}}$ Alexander Kaltenborn ${ }^{\mathrm{a}}$ Jürgen Klempnauer ${ }^{\mathrm{a}}$ Ulf Kulik $^{\mathrm{a}}$ \\ ${ }^{a}$ General, Visceral and Transplantation Surgery, Hannover Medical School, Hannover, Germany; ${ }^{b}$ Gastroenterology, \\ Hepatology and Endocrinology, Hannover Medical School, Hannover, Germany; ' Pathology, Hannover Medical \\ School, Hannover, Germany; ${ }^{d}$ Division of Transplant Surgery, Department of Surgery, Medical University of Graz, \\ Graz, Austria; 'Departament d'Astronomia i Astrofísica, Universitat de València, Valencia, Spain
}

\section{Keywords}

Colorectal cancer · Liver metastases - Liver resection · Chemotherapy · Steatosis

\begin{abstract}
Background: Hepatic steatosis and chemotherapy in the treatment of colorectal liver metastases (CLM) are often linked to increased mortality and morbidity after liver resection. This study evaluates the influence of macrovesicular hepatic steatosis and chemotherapeutic regimes on graded morbidity and mortality after liver resection for CLM. Methods: A total of 323 cases of liver resection for CLM were retrospectively analysed using univariable and multivariable linear, ordinal and Cox regression analyses. The resected liver tissue was re-evaluated by a single observer to determine the grade and type of hepatic steatosis. Results: Macrovesicular steatosis did not influence postoperative morbidity and survival, as evidenced by risk-adjusted multivariable Cox regression analysis ( $p=0.521)$. Conversion chemotherapy containing oxaliplatin was an independent and significant risk factor for mortality in risk-adjusted multivariable Cox regression analysis ( $p=0.005)$. Identified independently, significant risk factors for postoperative morbidity were neoadjuvant treatment of metastases of the primary tumour with irinotecan $(p=0.003)$, the duration of surgery in minutes $(p=0.001)$ and the number of intraoperatively transfused packed red blood cells ( $p \leq 0.001$ ). Surprisingly, macrovesicular hepatic steatosis was not a risk factor for postopera-
\end{abstract}

tive morbidity and was even associated with lower rates of complications $(p=0.006)$. Conclusion: The results emphasize the multifactorial influence of preoperative liver damage and chemotherapy on the severity of postoperative morbidity, as well as the significant impact of conversion chemotherapy containing oxaliplatin on survival.

(c) 2020 S. Karger AG, Basel

\section{Introduction}

Annually, 1.4 million patients are diagnosed with colorectal cancer worldwide; about $50 \%$ of these patients develop synchronous or metachronous liver metastases $[1,2]$. Surgical resection of colorectal liver metastases (CLM) is the treatment of choice, with 5-year survival rates of up to $50 \%$ [3-6]. Independent risk factors for survival after liver resection for CLM include limited liver function and pre-existing hepatocellular damage, transfusion requirements, the size of the largest metastasis and its distance to the resection margin [6].

Although survival rates have significantly improved during the last decades, the rate of postoperative complications (morbidity) remains relatively high at up to $38 \%$ $[7,8]$. Postoperative morbidity depends on common preoperative risk factors such as cardiovascular diseases, diabetes and ASA score, as well as surgery-specific risk factors such as the extent of surgery or the amount of transfused red blood cells [9]. Additionally, fatty liver de- 
generation is expected to become a major concern in liver surgery [10]. Obesity, a risk factor for developing colorectal cancer, is also associated with the development of non-alcoholic fatty liver disease [11]. Non-alcoholic fatty liver disease occasionally advances to a non-alcoholic steatohepatitis, and in some cases to a non-alcoholic steatohepatitis-related liver cirrhosis with severe reduction of liver function. As a consequence, surgical options in the treatment of CLM might be limited, mainly due to higher demands on the size of the liver remnant and impaired hepatic regeneration after liver resection.

However, not only obesity is considered as a risk factor for hepatic steatosis and a potential challenge in the surgical treatment of CLM. Increasing numbers of patients are receiving various regimes of chemotherapy prior to the resection of CLM, either with adjuvant intent for treatment of the primary colorectal lesion or as conversion therapy in case of primarily irresectable hepatic lesions. In this context, various chemotherapeutical agents are known to cause drug-specific hepatic toxicity [12]. While oxaliplatin is known for its association with sinusoidal obstruction syndrome (SOS), irinotecan is linked to chemotherapy-associated steatohepatitis, causing an increase in morbidity and mortality $[13,14]$.

Only few studies have evaluated the impact of hepatic steatosis or associated chemotherapies on the postoperative outcome after liver resection for CLM. Kurmann et al. [15] associated hepatic steatosis with surgical site infections after hepatic and colorectal surgery. Vauthey et al. [16] reported that steatohepatitis caused by chemotherapy increases the risk of 90-day mortality after liver resection for CLM. Veteläinen et al. [17] concluded that hepatic steatosis is a major risk factor for surgical complications and 60-day mortality. The influence of the type of steatosis that is, micro- or macrovesicular fat deposits - on morbidity and mortality after liver resection of CLM is mainly unclear. Data derived from orthotopic liver transplantations suggest that the degree of macrovesicular steatosis in the allograft is highly predictive of 90-day mortality [18].

The aim of this study was to investigate the influence of hepatic macrovesicular steatosis and chemotherapeutical regimes on patient survival and early morbidity, graded according to Dindo et al. [10], after liver resection for CLM.

\section{Patients and Methods}

\section{Data Collection}

This is a single-centre retrospective analysis with data from a German tertiary referral centre for hepatobiliary surgery and liver transplantation. The postoperative observational period ended on January 1st, 2017. Included were all cases of primary liver resection for CLM between January 1st, 2000, and October 31st, 2014, with available histological slides of the resection specimen. A descrip- tive summary of the cohort is provided in online supplementary Figure 1 and Tables 1 and 2 (for all online suppl. material, see www. karger.com/doi/10.1159/000510661).

Determination of Steatosis in Liver Resection Specimens

For the determination of hepatic steatosis, the same histological methodology was employed as described by Schrem et al. [18]. The extent of steatosis was assessed in the normal liver using histological slides from the liver resection specimen. The investigator was trained with pre-existing teaching slides by an expert in hepatic pathology. The first $20 \%$ were double-checked by that expert in a blinded fashion. Due to the fact that no mentionable differences occurred, the rest of the slides were assessed by a single observer with the pathologist in standby and ongoing random checks.

\section{Determination of Data on Chemotherapy}

Data on chemotherapy were gathered primarily from patients' medical charts. Additional information was collected on 313 patients using questionnaires sent to external oncologists and general practitioners who had treated the patients prior to liver resection. All data obtained were subjected to a series of automated plausibility checks as well as to scrutiny by an expert in gastroenterological oncology. Chemotherapies that had been applied for more than 6 months prior to CLM resection and resulted in resectable CLM that had initially been judged as irresectable were considered as conversion chemotherapy. The chemotherapeutic regimens were categorized according to the curative intent at the time of administration and according to the combinations of substances applied. Classified chemotherapeutic treatments that had been used in less than 8 cases prior to liver resection were subsumed under "other chemotherapeutic treatments" to enable meaningful statistical analysis.

\section{Study Endpoints}

The endpoints in this study were defined as patient survival in years, postoperative morbidity graded according to Dindo et al. [10] and the percentage of macrovesicular hepatic steatosis of normal liver tissue of the resection specimen.

\section{Statistical Methods}

Macrovesicular steatosis (\%) and variables to describe categorized chemotherapeutic treatments prior to liver resection were investigated to determine their independent influence on patient survival in Cox regression analysis, risk adjusted for previously identified independent risk factors for survival [6]. Risk factors for the percentage of macrovesicular hepatic steatosis and graded postoperative morbidity according to Dindo et al. [10] were assessed using linear and ordinal regression analysis, respectively.

Continuous variables with multicollinearity in correlation analysis with a factor correlation $R>|0.500|$ were subjected to a clinically informed decision on the exclusion of one of them, to avoid multicollinearity effects in regression modelling.

All variables that demonstrated an independently significant influence $(p<0.150$, the provided $p$ values correspond to two-sided probabilities) were then submitted to multivariable regression analysis in order to avoid overfitting [19]. Stepwise backwards likelihood elimination of the least significant variables $(p>0.050)$ was repeated until only significant variables remained in the regression model. These models enabled the identification of significant explanatory variables for all defined endpoints, as well as the quantification of the effect of each variable, that is, the degree and trend of their regression.

The software package JMP Pro 13 (SAS Institute, USA) was used to perform statistical analysis, as well as the vast, communitydriven and freely available infrastructure of the R-project to assess its usability as a cost-efficient alternative for upcoming studies. 
Table 1. Significant variables with influence on survival in Cox regression analysis

\begin{tabular}{|c|c|c|c|c|c|c|}
\hline \multirow[t]{2}{*}{ Variables } & \multicolumn{3}{|c|}{ Univariable regression } & \multicolumn{3}{|c|}{ Multivariable regression } \\
\hline & hazard ratio & $95 \%$ CI & $p$ value & hazard ratio & $95 \% \mathrm{CI}$ & $p$ value \\
\hline \multicolumn{7}{|l|}{ Preoperative details } \\
\hline BMI & 0.980 & $0.955-1.008$ & 0.144 & & & \\
\hline Haemoglobin (g/dL) & 0.851 & $0.774-0.935$ & 0.001 & & & \\
\hline Platelets $\left(\times 10^{3} / \mu \mathrm{L}\right)$ & 1.002 & $1.000-1.004$ & 0.068 & & & \\
\hline Quick (\%) & 0.988 & $0.977-0.998$ & 0.068 & 0.989 & $0.979-1.000$ & 0.048 \\
\hline Resection severity index & 1.102 & $1.015-1.196$ & 0.037 & & & \\
\hline \multicolumn{7}{|l|}{ Primary tumour characteristics } \\
\hline \multicolumn{7}{|l|}{ Location } \\
\hline Caecum & Reference & & & & & \\
\hline Colon ascendens & 0.610 & $0.329-1.128$ & & & & \\
\hline Colon transversum & 0.411 & $0.137-1.232$ & & & & \\
\hline Colon descendens & 0.391 & $0.177-0.865$ & 0.140 & & & \\
\hline Colon sigmoideum & 0.464 & $0.269-0.780$ & & & & \\
\hline Rectum & 0.497 & $0.291-0.849$ & & & & \\
\hline Neoadjuvant chemotherapy 5-FU & 0.605 & $0.358-1.023$ & 0.043 & & & \\
\hline Conversion chemotherapy 5-FU & 2.254 & $1.310-3.878$ & 0.009 & & & \\
\hline Conversion chemotherapy 5 -FU + oxaliplatin & 1.970 & $1.228-3.161$ & 0.010 & 2.074 & $1.252-3.436$ & 0.005 \\
\hline Conversion chemotherapy 5-FU + irinotecan & 1.767 & $1.197-2.608$ & 0.007 & & & \\
\hline Local relapse & 1.547 & $0.930-2.574$ & 0.114 & & & \\
\hline \multicolumn{7}{|l|}{ Operative details } \\
\hline Operative duration (min) & 1.003 & $1.001-1.004$ & 0.011 & & & \\
\hline Intraoperative transfusion of pRBCs & 1.850 & $1.408-2.432$ & $<0.001$ & 1.755 & $1.280-2.407$ & $<0.001$ \\
\hline Units of intraoperatively transfused pRBCs & 1.103 & $1.056-1.1521$ & $<0.001$ & & & \\
\hline Distance to resection margin $(\mathrm{mm})$ & 0.984 & $0.970-0.999$ & 0.018 & & & \\
\hline Size of largest metastases (mm) & 1.008 & $1.004-1.012$ & $<0.001$ & 1.006 & $1.002-1.010$ & 0.004 \\
\hline
\end{tabular}

BMI, body mass index; 5-FU, 5-fluorouracil; pRBCs, packed red blood cells.

\section{Results}

\section{Influence of the Categorized Chemotherapy Regimens} on Survival

As shown in Table 1 and Figure 2, only conversion chemotherapy containing 5-fluorouracil (5-FU) and oxaliplatin had a statistically significant independent influence on patient survival (HR 2.074; 95\% CI 1.252-3.436; $p=0.005)$.

\section{Variables with an Influence on Graded Postoperative}

Morbidity

The results of ordinal regression analysis are summarized in Table 2. Significant independent risk factors for graded postoperative morbidity until hospital discharge were neoadjuvant chemotherapeutic treatments of metastases containing irinotecan (OR 4.315; 95\% CI 1.751$10.633 ; p=0.003$ ), adjuvant chemotherapeutic treatments of the primary tumour containing irinotecan (OR 0.011; $95 \%$ CI $0-\infty ; p \leq 0.001)$, the duration of surgery in minutes (OR 1.007; 95\% CI 1.003-1.012; $p=0.001$ ) and the number of intraoperatively transfused packed red blood cells (pRBCs; OR 1.198; 95\% CI 1.077-1.332; $p \leq 0.001$ ). Surprisingly, macrovesicular hepatic steatosis showed a significant independent protective influence on the occurrence of postoperative complications (OR 0.965; 95\% CI 0.939-0.993; $p=0.006$ ).

\section{Risk Factors for Macrovesicular Steatosis}

The results of the linear regression analysis are summarized in Table 3. Independent risk factors for macrovesicular steatosis were body mass index $(p \geq 0.001)$, preoperative Quick ( $p=0.005)$ and conversion chemotherapeutic treatment with 5 -FU and oxaliplatin $(p=$ $0.003)$, as well as local relapse $(p=0.003)$ of the primary tumour and the size of the largest metastasis $(p=$ 0.006). To assess a pre-existing fibrosis, the FIB-4 score was included. An influence could not be shown in our cohort.

\section{Influence of Macrovesicular Steatosis on Survival}

The results of Cox regression analysis are summarized in Table 1. Separate multivariable Cox regression models revealed that macrovesicular steatosis (HR 0.997; 95\% CI $0.987-1.007 ; p=0.521$ ) did not show a statistically significant independent influence on patient survival. This result was confirmed in Kaplan-Meier analysis (shown in Fig. 1) using the log-rank test $(p=0.870)$. 
Table 2. Significant variables with influence on graded morbidity in ordinal regression analysis

\begin{tabular}{|c|c|c|c|c|c|c|}
\hline \multirow[t]{2}{*}{ Variables } & \multicolumn{3}{|l|}{ Univariable } & \multicolumn{3}{|c|}{ Multivariable } \\
\hline & odds ratio & $95 \% \mathrm{CI}$ & $p$ value & odds ratio & $95 \%$ CI & $p$ value \\
\hline \multicolumn{7}{|l|}{ Preoperative details } \\
\hline Haemoglobin (g/dL) & 0.769 & $0.638-0.927$ & 0.006 & & & \\
\hline Leucocytes $\left(\times 10^{3} / \mu \mathrm{L}\right)$ & 1.218 & $1.065-1.393$ & 0.005 & & & \\
\hline Platelets $\left(\times 10^{3} / \mu \mathrm{L}\right)$ & 1.005 & $1.001-1.008$ & 0.007 & & & \\
\hline Creatinine $(\mu \mathrm{mol} / \mathrm{L})$ & 0.985 & $0.967-1.003$ & 0.099 & & & \\
\hline Resection severity index & 1.154 & $0.976-1.366$ & 0.099 & & & \\
\hline \multicolumn{7}{|l|}{ Primary tumour characteristics } \\
\hline Neoadjuvant chemotherapy 5-FU + irinotecan & 3.999 & $1.762-9.076$ & 0.002 & 4.315 & $1.751-10.634$ & 0.003 \\
\hline Adjuvant chemotherapy 5-FU & 0.488 & $0.252-0.945$ & 0.026 & & & \\
\hline Adjuvant chemotherapy 5-FU + irinotecan & 0.000 & $0.000-\infty$ & 0.039 & $<0.001$ & $0.000-0.000$ & 0.011 \\
\hline \multicolumn{7}{|l|}{ Operative details } \\
\hline \multicolumn{7}{|l|}{1 point } \\
\hline Left atypical LR & & & & & & \\
\hline Right atypical LR & Reference & & & & & \\
\hline \multicolumn{7}{|l|}{2 points } \\
\hline \multicolumn{7}{|l|}{ Left segmental LR } \\
\hline Right segmental LR & 1.867 & $0.822-4.241$ & & & & \\
\hline \multicolumn{7}{|l|}{ Bilateral atypical LR } \\
\hline \multicolumn{7}{|l|}{3 points } \\
\hline Left hemihepatectomy & 0.413 & $0.051-3.371$ & & & & \\
\hline 4 points & & & 0.006 & & & \\
\hline Right hemihepatectomy & 2.016 & $0.827-4.913$ & & & & \\
\hline \multicolumn{7}{|l|}{5 points } \\
\hline Extended left hepatectomy & 6.197 & $2.404-15.976$ & & & & \\
\hline \multicolumn{7}{|l|}{6 points } \\
\hline Extended right hepatectomy & 2.957 & $0.980-8.925$ & & & & \\
\hline \multicolumn{7}{|l|}{7 points } \\
\hline Right hepatectomy + left atypical LR & 2.050 & $0.588-7.146$ & & & & \\
\hline Operative duration ( $\min$ ) & 1.010 & $1.006-1.013$ & $<0.001$ & 1.008 & $1.003-1.012$ & 0.001 \\
\hline Intraoperative transfusion of pRBCs & 4.213 & $2.369-7.491$ & $<0.001$ & & & \\
\hline Units of intraoperatively transfused pRBCs & 1.286 & $1.168-1.417$ & $<0.001$ & 1.198 & $1.077-1.332$ & $<0.001$ \\
\hline \multicolumn{7}{|l|}{ Metastasis grade } \\
\hline G1 & $<0.001$ & $0.000-0.000$ & & & & \\
\hline G2 & Reference & & 0.098 & & & \\
\hline G3 & $<0.001$ & $0.000-0.000$ & & & & \\
\hline \multicolumn{7}{|l|}{ R status } \\
\hline R0 & Reference & & & & & \\
\hline R1 & 3.532 & $1.324-9.423$ & 0.050 & & & \\
\hline R2 & 2.578 & $0.229-29.015$ & & & & \\
\hline Macrovesicular steatosis (\%) & 0.973 & $0.948-0.999$ & 0.023 & 0.965 & $0.939-0.993$ & 0.006 \\
\hline Size of largest metastases (mm) & 1.016 & $1.009-1.024$ & $<0.001$ & & & \\
\hline
\end{tabular}

LR, liver resection; pRBCs, packed red blood cells.

Figure 2 visualizes the most important findings. The full regression analyses are shown in online supplementary Tables 3, 4 and 5.

\section{Discussion}

This study was motivated by the increasing evidence for an impact of fatty liver disease on postoperative morbidity and survival after liver resection of CLM, especially in the context of preoperatively administered chemotherapies. The primary aim was to analyse the frequency and grade of macrovesicular steatosis in patients under- going liver resection for CLM and the chemotherapeutical regimes applied prior to surgery. Secondly, the potential influence of hepatic steatosis and associated chemotherapies on morbidity and survival after liver resection for CLM was explored.

Hepatic steatosis is mostly associated with secondary disorders such as the metabolic syndrome or other cardiovascular risk factors. Surprisingly, hepatic fat was not associated with a higher risk of postoperative morbidity in our study, and the body mass index showed no significant influence either in multivariable Cox regression analysis or in multivariable ordinal regression analysis. Patients with increasing degrees of liver fat deposits ap- 

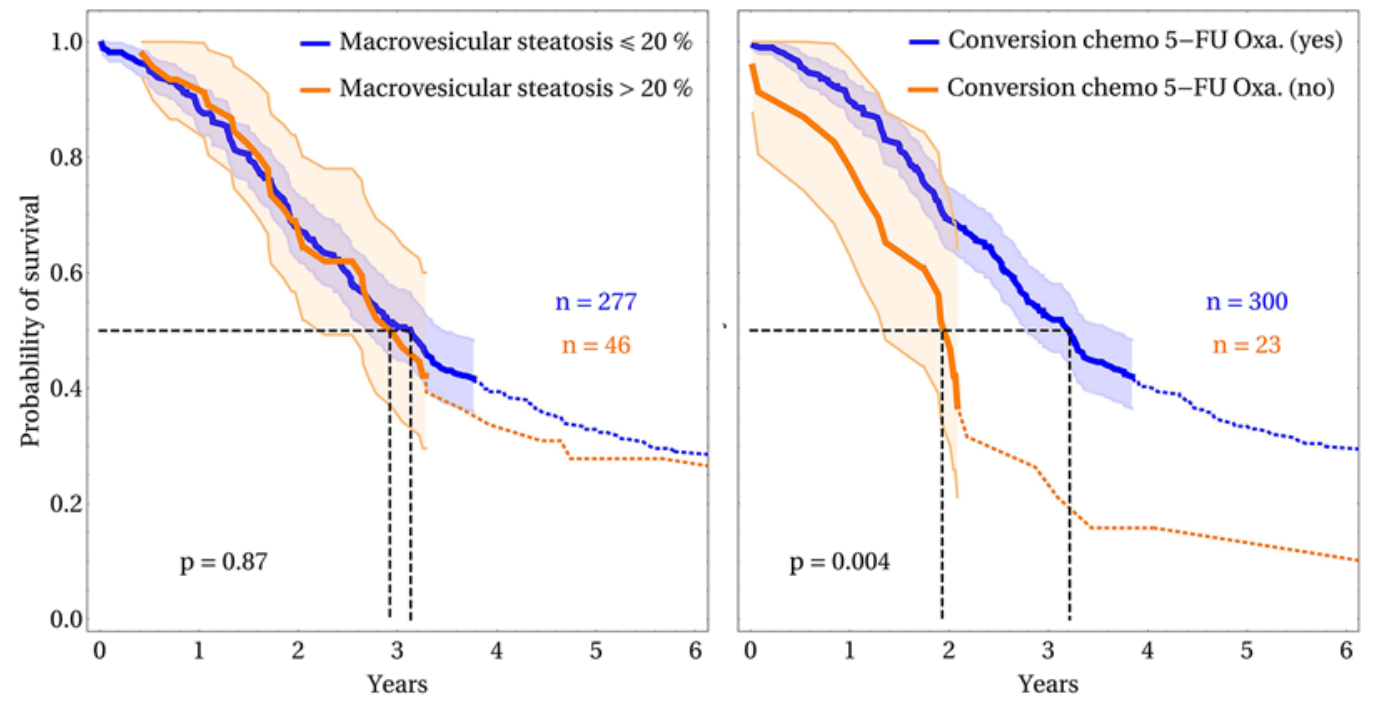

Fig. 1. Kaplan-Meier curves for macrovesicular steatosis and conversion chemotherapy with 5-fluorouracil (5FU) plus oxaliplatin (Oxa.). The $p$ values demonstrate the results of the log-rank test. The analyses were truncated when the numbers at risk were smaller than one-third of the starting number.

Table 3. Significant variables with influence on macrovesicular steatosis in linear regression analysis

\begin{tabular}{|c|c|c|c|}
\hline \multirow[t]{2}{*}{ Variables } & \multicolumn{2}{|c|}{ Univariable } & \multirow{2}{*}{$\begin{array}{l}\text { Multivariable } \\
p \text { value }\end{array}$} \\
\hline & $\overline{R^{2}}$ & $p$ value & \\
\hline \multicolumn{4}{|l|}{ Preoperative details } \\
\hline Age at resection of metastases (years) & 0.009 & 0.092 & \\
\hline Body mass index & 0.203 & $<0.001$ & $<0.001$ \\
\hline Haemoglobin (g/dL) & 0.043 & $<0.001$ & \\
\hline Quick (\%) & 0.028 & 0.003 & 0.005 \\
\hline Fibrosis-4 score & 0.013 & 0.077 & \\
\hline \multicolumn{4}{|l|}{ Primary tumour characteristics } \\
\hline Neoadjuvant chemotherapy 5-FU + irinotecan & 0.009 & 0.095 & \\
\hline Adjuvant chemotherapy 5-FU + oxaliplatin & 0.008 & 0.105 & \\
\hline Conversion chemotherapy 5 -FU & 0.012 & 0.049 & \\
\hline Conversion chemotherapy $5-\mathrm{FU}+$ oxaliplatin & 0.018 & 0.016 & 0.003 \\
\hline Conversion chemotherapy 5-FU + irinotecan & 0.008 & 0.111 & \\
\hline Local relapse & 0.018 & 0.016 & 0.003 \\
\hline \multicolumn{4}{|l|}{ Operative details } \\
\hline Intraoperative transfusion of pRBCs & 0.024 & 0.005 & \\
\hline Units of intraoperatively transfused pRBCs & 0.013 & 0.042 & \\
\hline Size of largest metastases $(\mathrm{mm})$ & 0.031 & 0.002 & 0.006 \\
\hline
\end{tabular}

pRBCs, packed red blood cells.

pear to have a lower risk of developing postoperative complications than patients without hepatic steatosis. This result contradicts previously published studies that reported increased morbidity in case of fatty liver disease $[20,21]$. Speculatively, the patients with hepatic steatosis in our cohort had undetected positive confounders explaining the lower rates of postoperative morbidity. Nev- ertheless, some other studies have suggested only a mild impact of steatosis on patient mortality and morbidity [17].

As determined by a one-tailed $z$ test, in order to quantitatively validate hepatic fat as a risk factor, a sample size of 10,000-100,000 patients would be needed. Of course, this exceeds the size of our study cohort, but in compari- 
Fig. 2. The figure visualizes the influence of relevant variables in multivariable regression analysis on morbidity and mortality. 5-FU, 5-fluorouracil; pRBCs, packed red blood cells.

\section{Significant variables with influence on survival} (multivariable cox regression)

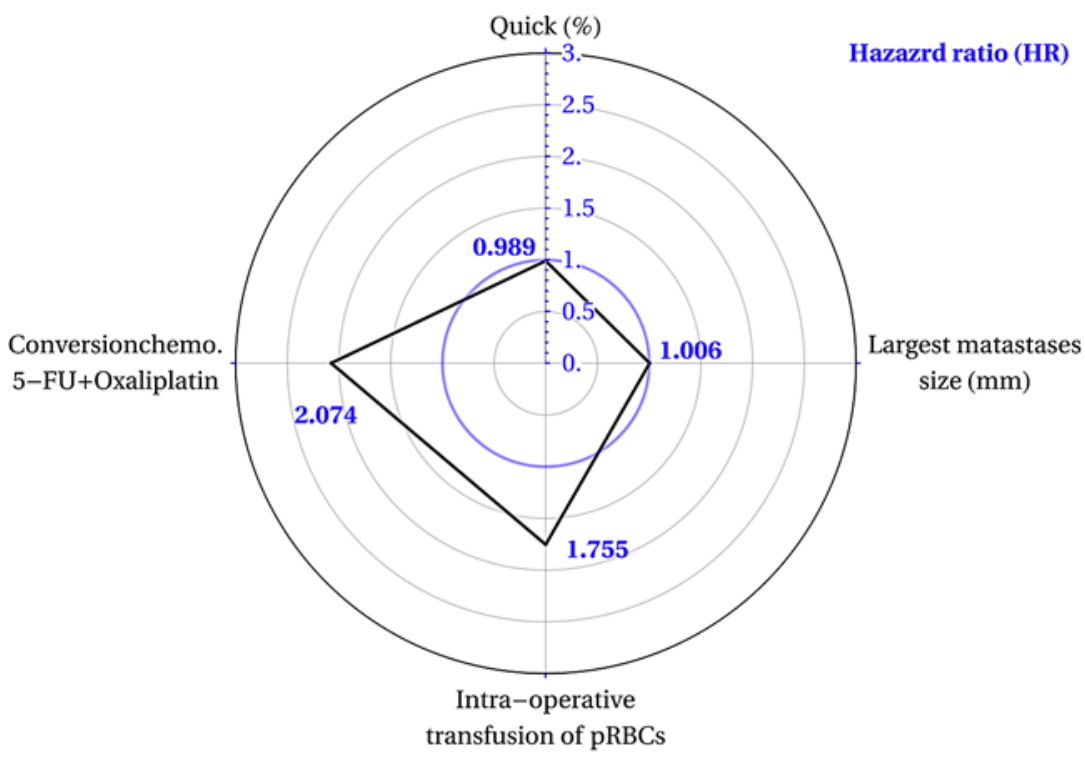

Significant variables with influence on graded morbidity (multivariable ordinal regression)

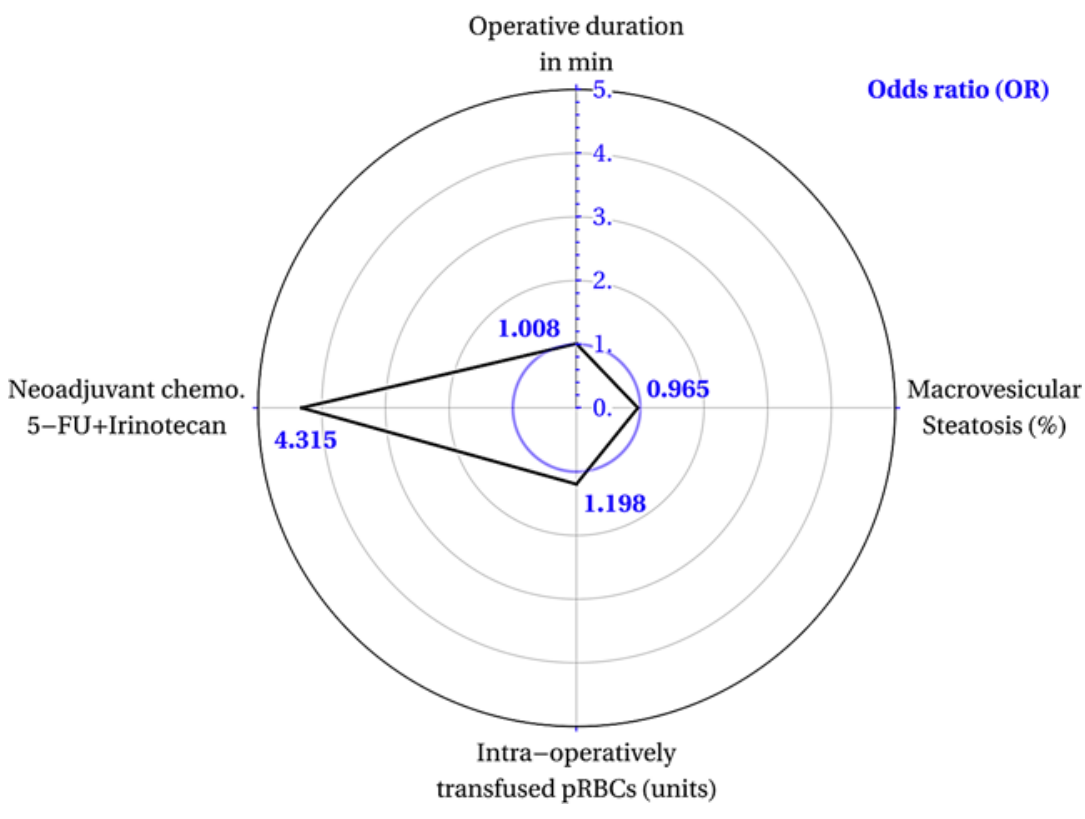

son to many recent studies, it appears of a reasonable size, especially when the additional information regarding chemotherapies is considered. Prospective studies are desirable to better understand the impact of steatosis on postoperative complications after liver resection for CLM, including cardiovascular risk factors or other secondary diseases. Moreover, as evidenced in our study, hepatic fat also had no influence on overall survival after resection of CLM. Hence, no negative effects of macrovesicular hepatic steatosis on survival (as reported for fatty liver allografts in the context of liver transplantations) could be confirmed in our analysis of liver resections for CLM [18].

For the first time, we demonstrated an independent influence of irinotecan on graded postoperative morbidity after liver resection for CLM (OR 4.315; 95\% CI 1.751- 
$10.633 ; p=0.003)$. This effect appears not to be related to drug-induced hepatic steatosis, since no influence of irinotecan on macrovesicular steatosis was observed in our cohort, and, as pointed out, steatosis did not influence morbidity. Nevertheless, irinotecan has been reported to cause chemotherapy-associated steatohepatitis [13, 14]. Possibly, in the case of irinotecan, inflammatory "second hits" are required to drive any increased morbidity of fatty livers after hepatic surgery. In fact, our study emphasizes the influence of oxaliplatin- and 5-FU-based chemotherapies on macrovesicular hepatic steatosis to highlight the hepatotoxicity of the substance and the association of oxaliplatin with SOS, as reported previously by Pessaux et al. [13].

Collectively, our findings underline that chemotherapeutical treatments prior to liver resection imply the possibility of chemotherapy-associated liver injury, resulting in increased postoperative morbidity [22]. In case of conversion chemotherapy containing 5-FU and oxaliplatin, an independent and statistically significant negative influence on patient survival was detectable. That effect might also be explained by possible oxaliplatin-based side effects such as polyneuropathy or decreased liver function caused by SOS. Even more importantly, conversion chemotherapy is probably only administered in case of an initially extensive and irresectable hepatic tumour burden, likely related to impaired overall survival. Hence, the liver resections were only possible after successful chemotherapeutical treatment, and our findings do not disparage the use of chemotherapy to achieve secondary resectability of CLM. Instead, surgeons must be aware of higher postoperative risks and possibly an impaired overall outcome, which is to be considered when counselling patients prior to surgical treatment. However, in case of a primary resectable CLM, the results emphasize that surgery should be considered whenever possible to avoid chemotherapy-induced liver injury.

In addition to the negative effects of chemotherapeutical agents on morbidity, the duration of surgery and the amount of transfusion requirements also appear to significantly increase the risk of postoperative complications. The time demand of the surgical procedure is mainly influenced by the experience of the surgeon and the extent of liver resection, but also by anatomical variables including the presence or absence of obesity. Therefore, the duration of resection might serve as a surrogate parameter for other factors that increase the risk of complications. Compatible with the higher rate of complications observed in case of longer surgical procedures, another recently published study reported that prolonged operative times caused higher rates of infection-related complications [23]. Furthermore, according to Gruttadauria et al. [24], the surgical time demand also correlates with intraoperative blood loss. In that study, intraopera- tive transfusion of pRBCs also increased the risk of complications of Clavien-Dindo grade III or higher. Our present study emphasizes that transfused pRBCs are an independently significant risk factor for higher grades of postoperative morbidity (OR 1.198; 95\% CI 1.077-1.332; $p \leq 0.001)$, although the correlation of operating time demand with pRBC requirements was low $(R=0.342)$. As reported previously, transfusion requirements depend on the extent of liver resection, the proportional frequency of anatomical resections and the prolonged use of portal triad clamping [23]. Additionally, several studies have demonstrated blood loss to be an independent risk factor for compromised short-term and long-term outcome after liver resection [23, 25-28]. A correlation between extent of steatosis and extent of resection, possibly acting as a selection bias, could not be detected.

The sample size of our cohort might be considered as a limitation of the study. Nevertheless, other studies suggest a mild impact of hepatic steatosis on patient mortality and morbidity [17]. As determined by a one-tailed $z$ test, in order to quantitatively validate hepatic steatosis as an individual risk factor, a sample size of 10,000-100,000 patients would be needed. Such sample sizes exceed the cohorts of all trials considered and the possibilities of single-centre analyses.

\section{Conclusions}

An increased risk of the development of postoperative complications or impaired overall survival caused by hepatic steatosis could not be evidenced. We highlight the association of irinotecan with higher rates of postoperative complications. A conversion chemotherapy containing oxaliplatin and 5-FU was associated with limited overall survival, most likely referring to more extensive hepatic tumour disease. It must be marked that the clinical usefulness in preoperative patient counselling is limited, since most of the variables that appear to influence postoperative morbidity are only available after the performance of surgery, that is, duration and $\mathrm{pRBC}$ demand. Preoperative imaging occasionally indicates signs of hepatic steatosis, and even the duration of surgery and expectable blood loss can be anticipated based on the extent of hepatic segments affected by CLM. Hence, an approximate identification of potential patients at risk is still reasonable, eventually allowing for more profound patient counselling and intensified postoperative surveillance. 


\section{Statement of Ethics}

The institution's Ethics Committee approved this retrospective study (Ethikkommission, Hannover Medical School; approval decision No. 3079-2016). All patients provided informed consent. Patient data were anonymized and de-identified prior to analysis.

\section{Conflict of Interest Statement}

The authors have no conflicts of interest to declare.

\section{Funding Sources}

J.F.M. acknowledges the Grisolia Grant GRISOLIAP/2016/097 and a Ph.D. grant from the Studienstiftung des Deutschen Volkes.

\section{Author Contributions}

J.C.M.: data collection and interpretation, statistical analysis, histological assessment of steatosis and preparation of the manuscript; T.C.W.: evaluation of chemotherapies and revision of the manuscript; B.H.: teaching of assessment of steatosis and doublechecking of results, and revision of the manuscript; H.S. and J.F.M.: statistical analysis, interpretation of data and revision of the manuscript; A.K.: study design, statistical analysis, interpretation of data and revision of the manuscript; J.K.: study design and revision of the manuscript; U.K.: study design, statistical analysis, interpretation of data and preparation of the manuscript.

\section{References}

1 Kulik U, Bektas H, Klempnauer J, Lehner F. Repeat liver resection for colorectal metastases. Br J Surg. 2013 Jun;100(7):926-32.

2 Ferlay J, Soerjomataram I, Dikshit R, Eser S, Mathers C, Rebelo M, et al. Cancer incidence and mortality worldwide: sources, methods and major patterns in GLOBOCAN 2012. Int J Cancer. 2015 Mar;136(5):E359-86.

3 Gong Y, Liu Z, Liao Y, Mai C, Chen T, Tang $\mathrm{H}$, et al. Effectiveness of $\omega-3$ Polyunsaturated Fatty Acids Based Lipid Emulsions for Treatment of Patients after Hepatectomy: A Prospective Clinical Trial. Nutrients. 2016 Jun; 8(6):E357.

4 Hayashi K, Yokoyama Y, Nakajima H, Nagino $\mathrm{M}$, Inoue $\mathrm{T}$, Nagaya $\mathrm{M}$, et al. Preoperative 6-minute walk distance accurately predicts postoperative complications after operations for hepato-pancreato-biliary cancer. Surgery. 2017 Feb;161(2):525-32.

5 Chakedis J, Squires MH, Beal EW, Hughes T, Lewis H, Paredes A, et al. Update on current problems in colorectal liver metastasis. Curr Probl Surg. 2017 Nov;54(11):554-602.

6 Gwiasda J, Schrem H, Kaltenborn A, Mahlmann J, Mix H, Lehner F, et al. Introduction of the resection severity index as independent risk factor limiting survival after resection of colorectal liver metastases. Surg Oncol. 2017 Dec;26(4):382-8.

7 Shinke G, Noda T, Eguchi H, et al. Surgical outcome of extended liver resections for colorectal liver metastasis compared with standard liver resections. Mol Clin Oncol. 2018 Jul;9(1):104-11.

8 Levy M, Lipska L, Mracek M, Jirsova A, Simsa J, Visokai V. Postoperative complications after liver resection for colorectal metastases. HPB. 2016;18:e685-6.

9 Andres A, Toso C, Moldovan B, Schiffer E, Rubbia-Brandt L, Terraz S, et al. Complications of elective liver resections in a center with low mortality: a simple score to predict morbidity. Arch Surg. 2011 Nov;146(11):1246-52.

10 Dindo D, Demartines N, Clavien PA. Classification of surgical complications: a new proposal with evaluation in a cohort of $6,336 \mathrm{pa}-$ tients and results of a survey. Ann Surg. 2004 Aug;240(2):205-13.

11 Calle EE, Rodriguez C, Walker-Thurmond K, Thun MJ. Overweight, obesity, and mortality from cancer in a prospectively studied cohort of U.S. adults. N Engl J Med. 2003 Apr; 348(17):1625-38.

12 Passot G, Soubrane O, Giuliante F, Zimmitti G, Goéré D, Yamashita S, et al. Recent Advances in Chemotherapy and Surgery for Colorectal Liver Metastases. Liver Cancer. 2016 Nov;6(1):72-9.

13 Pessaux P, Chenard MP, Bachellier P, Jaeck D. Consequences of chemotherapy on resection of colorectal liver metastases. J Visc Surg. 2010 Aug;147(4):e193-201.

14 Morris-Stiff G, Tan YM, Vauthey JN. Hepatic complications following preoperative chemotherapy with oxaliplatin or irinotecan for hepatic colorectal metastases. Eur J Surg Oncol. 2008 Jun;34(6):609-14.

15 Kurmann A, Wanner B, Martens F, Klasen J, Stickel F, Montani M, et al. Hepatic steatosis is associated with surgical-site infection after hepatic and colorectal surgery. Surgery. 2014 Jul;156(1):109-16.

16 Vauthey JN, Pawlik TM, Ribero D, Wu TT, Zorzi D, Hoff PM, et al. Chemotherapy regimen predicts steatohepatitis and an increase in 90-day mortality after surgery for hepatic colorectal metastases. J Clin Oncol. 2006 May; 24(13):2065-72.

17 Veteläinen $\mathrm{R}$, van Vliet A, Gouma DJ, van Gulik TM. Steatosis as a risk factor in liver surgery. Ann Surg. 2007 Jan;245(1):20-30.

18 Schrem H, Focken M, Gunson B, Reichert B, Mirza D, Kreipe HH, et al. The new liver allocation score for transplantation is validated and improved transplant survival benefit in Germany but not in the United Kingdom. Liver Transpl. 2016 Jun;22(6):743-56.

19 Hosmer D, Lemeshow S, May S. Applied survival analysis: regression modeling of timeto-event data. 2 nd edition. Hoboken, NJ: Wiley-Interscience; 2008.

20 Belghiti J, Hiramatsu K, Benoist S, Massault $\mathrm{P}$, Sauvanet A, Farges O. Seven hundred forty- seven hepatectomies in the 1990s: an update to evaluate the actual risk of liver resection. J Am Coll Surg. 2000 Jul;191(1):38-46.

21 Zorzi D, Laurent A, Pawlik TM, Lauwers GY Vauthey JN, Abdalla EK. Chemotherapy-associated hepatotoxicity and surgery for colorectal liver metastases. Br J Surg. 2007 Mar;94(3):274-86.

22 Lock JF, Westphal T, Rubin T, Malinowski M, Schulz A, Jara M, et al. LiMAx Test Improves Diagnosis of Chemotherapy-Associated Liver Injury before Resection of Colorectal Liver Metastases. Ann Surg Oncol. 2017 Sep;24(9): 2447-55.

23 Gupta R, Fuks D, Bourdeaux C, Radkani P, Nomi T, Lamer C, et al. Impact of intraoperative blood loss on the short-term outcomes of laparoscopic liver resection. Surg Endosc. 2017 Nov;31(11):4451-7.

24 Gruttadauria S, Saint Georges Chaumet M, Pagano D, Marsh JW, Bartoccelli C, Cintorino $\mathrm{D}$, et al. Impact of blood transfusion on early outcome of liver resection for colorectal hepatic metastases. J Surg Oncol. 2011 Feb; 103(2):140-7.

25 Ejaz A, Spolverato G, Kim Y, Margonis GA, Gupta R, Amini N, et al. Impact of blood transfusions and transfusion practices on long-term outcome following hepatopancreaticobiliary surgery. J Gastrointest Surg. 2015 May;19(5):887-96.

26 Jarnagin WR, Gonen M, Fong Y, DeMatteo $\mathrm{RP}$, Ben-Porat L, Little S, et al. Improvement in perioperative outcome after hepatic resection: analysis of 1,803 consecutive cases over the past decade. Ann Surg. 2002 Oct;236(4): 397-406.

27 Margonis GA, Kim Y, Samaha M, Buettner S, Sasaki K, Gani F, et al. Blood loss and outcomes after resection of colorectal liver metastases. J Surg Res. 2016 May;202(2):47380.

28 Schiergens TS, Stielow C, Schreiber S, Hornuss C, Jauch KW, Rentsch M, et al. Liver resection in the elderly: significance of comorbidities and blood loss. J Gastrointest Surg. 2014 Jun;18(6):1161-70. 